\title{
Dahuang Zexie Decoction Protects against High-Fat Diet-Induced NAFLD by Modulating Gut Microbiota-Mediated Toll-Like Receptor 4 Signaling Activation and Loss of Intestinal Barrier
}

\author{
Jing Fang, ${ }^{1}$ Xiaoqi Sun, ${ }^{2}$ Boyu Xue, ${ }^{1}$ Nanyuan Fang, ${ }^{1,3}$ and Min Zhou ${ }^{1,3}$ \\ ${ }^{1}$ The First College of Clinical Medicine, Nanjing University of Chinese Medicine, Nanjing 210023, China \\ ${ }^{2}$ Department of Police Tactics, Nanjing Forest Police College, Nanjing 210023, China \\ ${ }^{3}$ Department of Infectious Disease, Jiangsu Province Hospital of Traditional Chinese Medicine, Affiliated Hospital of \\ Nanjing University of Chinese Medicine, Nanjing 210029, China
}

Correspondence should be addressed to Min Zhou; zhoumdoctor@163.com

Received 20 July 2017; Revised 24 September 2017; Accepted 4 October 2017; Published 12 November 2017

Academic Editor: Chang G. Son

Copyright (c) 2017 Jing Fang et al. This is an open access article distributed under the Creative Commons Attribution License, which permits unrestricted use, distribution, and reproduction in any medium, provided the original work is properly cited.

Increasing evidence suggests that intestinal dysbiosis, intestinal barrier dysfunction, and activated Toll-like receptor 4 (TLR4) signaling play key roles in the pathogenesis of NAFLD. Dahuang Zexie Decoction (DZD) has been verified to be effective for treating NAFLD, but the mechanisms remain unclear. In this study, we investigated the effects of DZD on NAFLD rats and determined whether such effects were associated with change of the gut microbiota, downregulated activity of the TLR4 signaling pathway, and increased expressions of tight junction (TJ) proteins in the gut. Male Sprague Dawley rats were fed high-fat diet (HFD) for 16 weeks to induce NAFLD and then given DZD intervention for 4 weeks. We found that DZD reduced body and liver weights of NAFLD rats, improved serum lipid levels and liver function parameters, and relieved NAFLD. We further found that DZD changed intestinal bacterial communities, inhibited the intestinal TLR4 signaling pathway, and restored the expressions of TJ proteins in the gut. Meanwhile ten potential components of DZD had been identified. These findings suggest that DZD may protects against NAFLD by modulating gut microbiota-mediated TLR4 signaling activation and loss of intestinal barrier. However, further studies are needed to clarify the mechanism by which DZD treats NAFLD.

\section{Introduction}

Nonalcoholic fatty liver disease (NAFLD), as the hepatic manifestation of metabolic syndrome [1], is typified by fat accumulation in the liver without significant alcohol consumption [2]. Recently, NAFLD has become the most common cause for chronic liver disease worldwide [3-5]. The prevalence rate of NAFLD is $25.24 \%$ globally, being highest in the Middle East and South America and lowest in Africa. NAFLD has been associated with obesity, type 2 diabetes mellitus, hyperlipidemia, hypertension, fibrosis, and hepatocellular carcinoma [6]. However, the pathogenesis of NAFLD has not been completely clarified. It is wellestablished that the gut microbiota is significantly involved in the pathogenesis of NAFLD $[7,8]$. The gut is open to the outer environment, and the gut microbiota is a complex microbial community inhabiting the intestinal tract that includes 100 trillion bacteria with over 1000 species. It contains genetic materials severalfold those of human genome and produces considerable metabolites and peptides. Accumulating evidence has proven that NAFLD increased energy harvesting upon intestinal dysbiosis or bacterial overgrowth $[9,10]$. Patients with NAFLD have lower percentages of Bacteroidetes and Ruminococcaceae than those in normal subjects $[11,12]$. Besides, damage of the intestinal barrier also significantly contributes to NAFLD. The intestinal mucosal mechanical barrier is the first defense of intestinal barrier, which is composed of intestinal epithelial cells (IECs), tight junction (TJ), and the mucous layer that covers the surface of IECs. TJ plays a crucial role in maintaining the integrity of the 
intestinal barrier, which promotes nutrient and water transport and also protects against gut-derived pathogens [13]. In the case of NAFLD, intestinal bacterial overgrowth generates gut-derived pathogens such as endotoxin or lipopolysaccharide (LPS) which are the main components of cell walls in Gram-negative bacteria. Meanwhile, they can activate the Toll-like receptor 4 (TLR4) signaling pathway. After binding TLR4 on the cell membrane, they increase the intestinal permeability by downregulating TJ proteins. As we all know, the liver is located in the proximity of the gut, receiving $75 \%$ of its blood supply through the portal vein. The portal vein flow not only carries nutrients, but also translocates microbial products and bacteria. Due to increased intestinal permeability (leaky gut), gut-derived pathogens can penetrate the intestinal barrier into the portal vein and cause NAFLD by inducing liver inflammation and fat deposition.

In Western countries, there is still no proven medical therapy for NAFLD hitherto. In contrast, traditional Chinese medicine drugs have exhibited remarkable therapeutic effects on NAFLD. Dahuang Zexie Decoction (DZD), as a Chinese herbal formula, consists of three herbs, Zexie (Alisma orientale), Baizhu (Atractylodes macrocephala), and Dahuang (Rheum palmatum). It was developed from Zexie Decoction, a classical prescription documented in Synopsis of the Golden Chamber, which was completed in the Chinese Han dynasty (206 BC-220 AD). Zexie Decoction only has two herbs, Zexie (A. orientale) and Baizhu (A. macrocephala). It can obviously mitigate NAFLD and decrease blood lipid levels [14]. Thereby motivated, we established a rat model of high-fat diet- (HFD-) induced NAFLD and then evaluated the effects of DZD on the liver function and gut microbiota, together with the expressions of intestinal TJ proteins and members in the TL4 signaling pathway in these rats.

\section{Materials and Methods}

2.1. Preparation of DZD. We prepared DZD by a mixture comprising the following three dried herbs: Zexie (Alisma orientalis, $30 \mathrm{~g}$ ), Baizhu (Atractylodes macrocephala, $12 \mathrm{~g}$ ), and Dahuang (Rheum palmatum, $15 \mathrm{~g}$ ). The herbs were extracted with water, concentrated to the density of $1 \mathrm{~g}$ crude herb/ml, and stored at $-20^{\circ} \mathrm{C}$ until further use. All the herbal components were purchased from Baicaotang Outpatient Department, Nanjing University of Chinese Medicine (Nanjing, China). Herbs were obtained from qualified suppliers on the basis of standards specified in the Chinese Pharmacopoeia (2010 Edition).

2.2. Animals. The protocols for animal studies were reviewed and approved by the Animal Studies Ethics Committee of Nanjing University of Chinese Medicine. Eight-week-old male Sprague Dawley (SD) rats $(n=24)$ were housed in a controlled environment ( $12 \mathrm{~h}-12 \mathrm{~h}$ light-dark cycle) in the Animal Center of Nanjing University of Chinese Medicine. After one week of acclimation on normal diet, the rats were randomly divided into three groups and fed either normal diet or HFD. Normal diet (fat contributed $10 \%$ calories) and HFD (fat contributed $45 \%$ calories) were purchased from Jiangsu Medicine Biological \& Pharmaceutical Co., Ltd.
(Yangzhou, China). A control group was fed normal diet for 16 weeks $(n=8)$, and the remaining sixteen rats were fed HFD for 16 weeks. After 16 weeks of HFD feeding, the sixteen rats were further divided into 2 groups: NAFLD group and DZD group $(n=8)$. The DZD group received daily gavage of $5.13 \mathrm{~g} / \mathrm{kg}$ DZD (density: $1 \mathrm{~g}$ crude herb/ml) in addition to HFD for four weeks, while the NAFLD group was gavaged with the same volume of saline for four weeks. The control group was fed normal diet and saline for four weeks. Body weight gain was assessed once a week. After 4 weeks of gavage, the animals were anesthetized with $4 \%$ chloral hydrate and then sacrificed. Blood was drawn and collected into tubes. Liver tissue was weighed and collected. Intestine and feces samples were also collected.

2.3. Reagents. Antibody against TLR4 was purchased from Abnova (TW, China). Antibodies against myeloid differentiation factor 88 (MyD88), $p$-JNK, JNK, p-ERK, and ERK were purchased from Cell Signaling Technology (MA, USA). Antibody against occludin and goat anti-mouse secondary antibody were purchased from Abcam (MA, USA). Antibody against zonula occludens-1 (ZO-1) was purchased from Thermo Fisher Scientific (MA, USA). Antibody against $\beta$ actin and goat anti-rabbit secondary antibody were purchased from Santa Cruz Biotechnology (CA, USA).

2.4. Biochemical Assays. Triglyceride (TG), total cholesterol (TC), low density lipoprotein-cholesterol (LDL-C), high density lipoprotein-cholesterol (HDL-C), alanine aminotransferase (ALT), and aspartate aminotransferase (AST) in serum were measured by commercial kits (Nanjing Jiancheng Institute of Biotechnology, Nanjing, China) according to the manufacturer's instructions.

2.5. Histopathological Examination. Liver pieces of about $5 \mathrm{~mm}$ in all dimensions were obtained from rats and fixed in $4 \%$ formaldehyde for $15 \mathrm{~min}$. Afterwards, the specimens were sequentially equilibrated in $30 \%$ sucrose, $15 \%$ sucrose $/ 50 \%$ optimal cutting temperature medium (OCT, Sakura Finetek, Torrance, CA, USA), and 100\% OCT. Liver pieces were subsequently frozen in OCT and $10 \mu \mathrm{m}$-thick sections were cut with a cryostat. The sections were then stained with hematoxylin/eosin (HE) and visualized by light microscopy.

2.6. Immunohistochemical Assay. Ileum and colon tissues were obtained to detect ZO-1 and occludin. The above sections were used for immunohistochemistry, and rehydrated before immunostaining. After blocking, the sections were incubated with rabbit anti-rat ZO-1 and occludin antibodies (Invitrogen, USA). The immunostaining results were reviewed and scored independently by two pathologists.

2.7. Western Blotting. The protein expressions of ZO-1, occludin, TLR4, MyD88, $p$-JNK, JNK, $p$-ERK, and ERK in the gut were detected by Western blotting. In brief, total protein was extracted from distal ileum and colon tissues, and the concentrations of supernatants were measured using the BCA protein assay (Thermo Fisher). Aliquots of supernatants containing $30 \mu \mathrm{g}$ of protein were electrophoresed on $10 \%(\mathrm{w} / \mathrm{v})$ 
sodium dodecyl sulfate-polyacrylamide gels and transferred onto polyvinylidene difluoride membranes. After blocking for $1 \mathrm{~h}$ with $5 \%(\mathrm{w} / \mathrm{v})$ skimmed milk, the membranes were incubated with primary antibodies at $4^{\circ} \mathrm{C}$ overnight and then washed by tris-buffered saline and incubated with secondary antibodies for $1 \mathrm{~h}$. Protein bands were analyzed with image analysis software Quantity one. The results were expressed as ratios relative to $\beta$-actin as the internal control.

\subsection{DNA Extraction, 16S Ribosomal RNA (rRNA) Gene} Sequencing, and Microbial Analysis of Fecal Samples. Fecal samples were collected and frozen at $-80^{\circ} \mathrm{C}$ within $3 \mathrm{~h}$ after sampling. DNA extraction was performed using a QIAamp Fast DNA Stool Mini kit (Qiagen, California, USA). Purity was determined and concentration was calculated. The V3 region of bacterial 16S rRNA gene was amplified by PCR. The bacterial genomic DNA was amplified by PCR with forward primer $\left(5^{\prime}\right.$-TCGTCGGCAGCGTCAGATGTGTATAAGAGACAGCCTACGGGNGGCWGCAG) and reverse primer (5'-GTCTCGTGGGCTCGGAGATGTGTATAAGAGACAGGACTACHVGGGTATCTAATCC) for the V3 hypervariable region. Purified amplicons were pooled into equimolar concentrations, and pairedend sequencing was performed using an Illumina MiSeq instrument (Illumina, San Diego, California, USA). The representative sequences of OTUs were used to analyze $\alpha$ diversity (Chao index and Shannon diversity index) on the basis of their relative abundances. A heatmap was generated according to the relative abundances of OTUs by R software (https://www.R-project.org). Phylogenetic $\beta$-diversity measures such as unweighted UniFrac significance test, principal coordinate analysis, and nonmetric multidimensional scaling were performed using the representative sequences of OTUs for each sample by the Mothur program to analyze community and phylogenesis. Taxonomy-based analyses were performed for taxonomic classification using the ribosomal database project classifier with a $60 \%$ bootstrap score.

\subsection{High-Performance Liquid Chromatography Coupled with} Mass Spectrometry (HPLC-MS). DZD water extract was analyzed by high-performance liquid chromatography with hybrid linear ion trap Orbitrap mass spectrometry (HPLCLTQ/Orbitrap) (Thermo Fisher Scientific, USA) with an Accucore C chromatographic column $(182.1 \mathrm{~mm} \times 150 \mathrm{~mm}$, $0.26 \mu \mathrm{m}$, Thermo Fisher Scientific, USA) and a $5 \mathrm{~cm}$ Lot 13375 guard column (Thermo Fisher Scientific, USA). The mobile phase consisted of (A) $100 \%$ acetonitrile and (B) $100 \%$ water with $0.1 \%$ formic acid. Gradient elution was conducted by using 5\%-12\% (0-30 min), 40\% (35 min), 40\% (52 $\mathrm{min}$ ), $70 \%$ (63 $\mathrm{min}), 85 \%$ (67 $\mathrm{min}$ ), and $85 \%$ acetonitrile (70 $\mathrm{min})$. The flow rate was $1 \mathrm{~mL} / \mathrm{min}$ and the detection range was full wavelength. The mass spectrometry (MS) method consisted of positive and negative ion detection model. The ESI source parameters were set as follows: ion spray voltage $2.4 \mathrm{kV}$, capillary temperature $300^{\circ} \mathrm{C}$, source heater temperature $110^{\circ} \mathrm{C}$, sheath gas (N2) 40 arbitrary units, auxiliary gas (N2) 10 arbitrary units, and sweep gas (N2) 0 arbitrary units. The Orbitrap analyzer scanned the mass with the range of $m / z$ 120-900.
2.10. Statistical Analysis. SPSS 18.0 and GraphPad Prism 5 were used for statistical analysis. Data were expressed as mean \pm standard error of mean (SEM). One-way analysis of variance with Tukey's correction was applied for differences between two groups, and $P<0.05$ was accepted as statistically significant.

\section{Results}

(1) Therapeutic Effect of DZD on Rat Model of HFD-Induced NAFLD. To evaluate the therapeutic effect of DZD on NAFLD in the HFD-fed rat model, we detected the body weight, liver weight, serum lipid levels, liver function parameters, and pathological changes of three groups. Compared to the control group, the HFD group had higher body weight, liver weight, and serum ALT, AST, TG, TC, HDL-C, and LDL-C levels (Figures $1(\mathrm{a})-1(\mathrm{~h})$ ). DZD treatment for 4 weeks significantly decreased body and liver weights and restored serum lipid levels and liver function parameters compared with those of the HFD group (Figures $1(\mathrm{a})-1(\mathrm{~h})$ ). Meanwhile, the body weight, liver weight, serum lipids, and liver function parameters of the DZD group were similar to those of the control group. The three groups also had similar serum glucose levels (Figure 1(i)). Consistently, the liver sections of the HFD group showed extensive macrosteatosis and hepatocyte ballooning which were relieved in those of DZD-treated rats (Figure $1(\mathrm{j})$ ). Collectively, DZD exerted beneficial effects on the NAFLD rat model.

(2) Effects of DZD Therapy on the Gut Microbiota Composition of NAFLD Rats. NAFLD is associated with intestinal dysbiosis, so we analyzed the effects of HDF and DZD on the gut microbiota composition. We used MiSeq technology to conduct bacterial $16 \mathrm{~S}$ rRNA sequencing after 4 weeks of treatment. The quality of sequencing, which included microbial richness and biodiversity, met the requirements for subsequent analysis. Both HFD and DZD decreased the Shannon index compared with that of the control group (Figure 2(a)), so they significantly reduced the diversity of gut microbiota. Meanwhile, the three groups had similar richness, as indicated by the Chao index (Figure 2(b)). Samples from the three groups formed distinct clusters in the ordination plot, suggesting that HFD and DZD induced main changes in the gut microbiota (Figure 2(c)). The HFD group had a higher relative abundance of Firmicutes and a lower relative abundance of Bacteroidetes than those of the control group, and DZD decreased the proportion of Firmicutes and increased that of Bacteroidetes on the phylum level (Figures 2(d) and 2(e)). Besides, principle component analysis (PCoA) was performed to compare the differences in bacterial communities between different groups on the OTU level (Figure 2(f)). In addition, changes of intestinal microbiota on the family level showed that Ruminococcaceae significantly decreased and Desulfovibrionaceae significantly increased in the HFD group compared to those in the control group (Figures 3(a) and 3(b)). In contrast, DZD administration restored these proportions (Figures 3(a) and 3(b)). On the genus level, the relative abundances of Desulfovibrio and Escherichia/Shigella were significantly higher in the NAFLD 


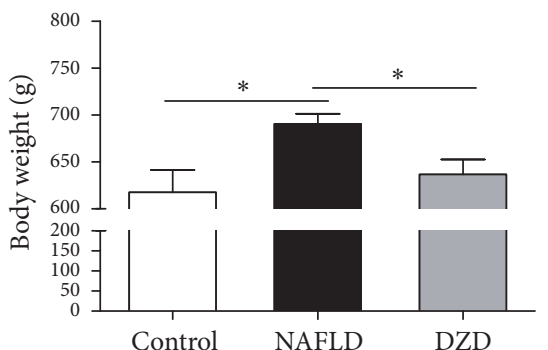

(a)

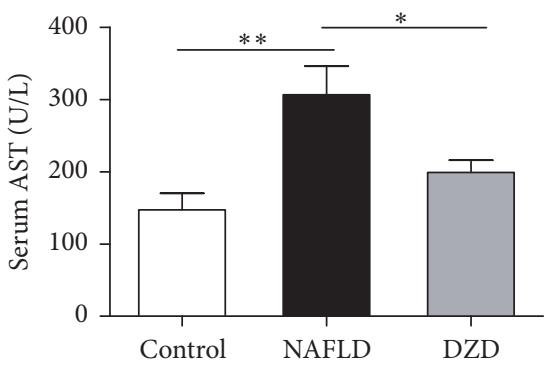

(d)

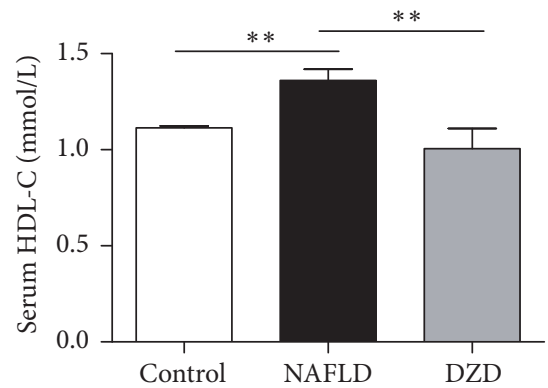

(g)

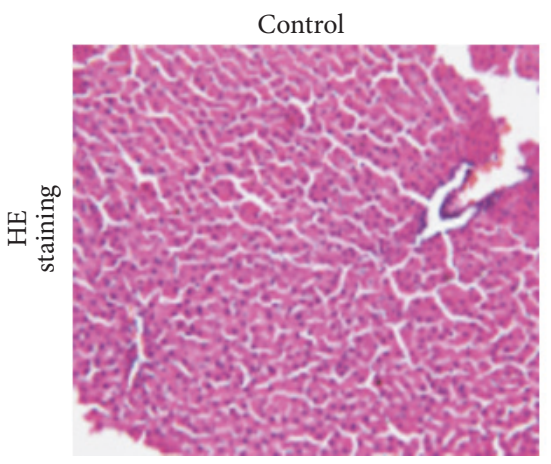

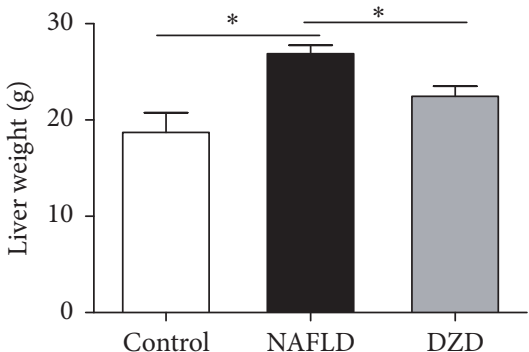

(b)

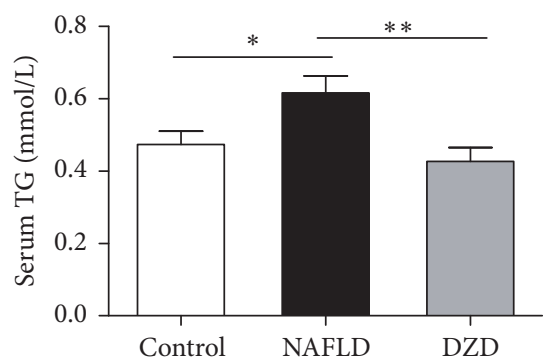

(e)

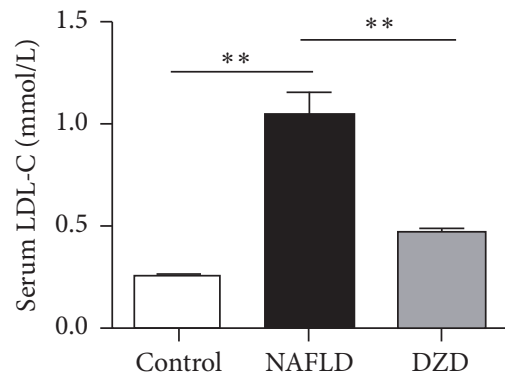

(h)

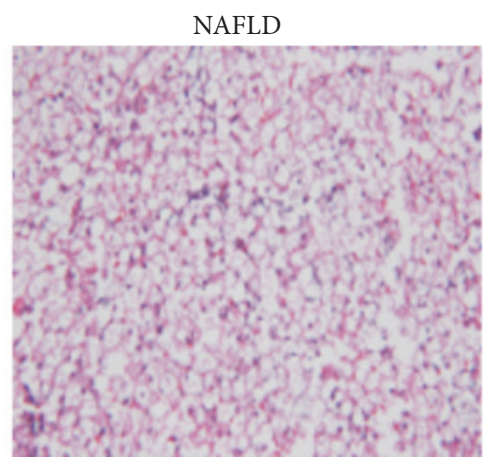

(j)

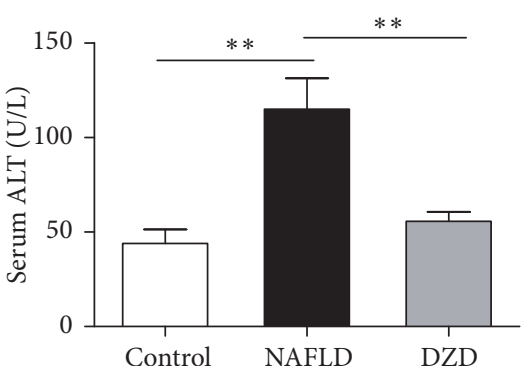

(c)

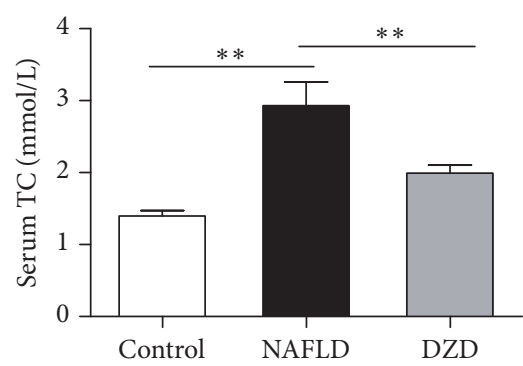

(f)

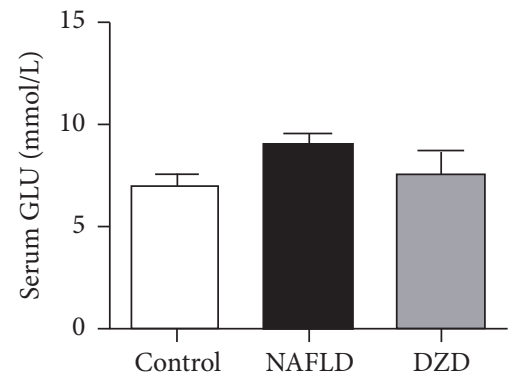

(i)

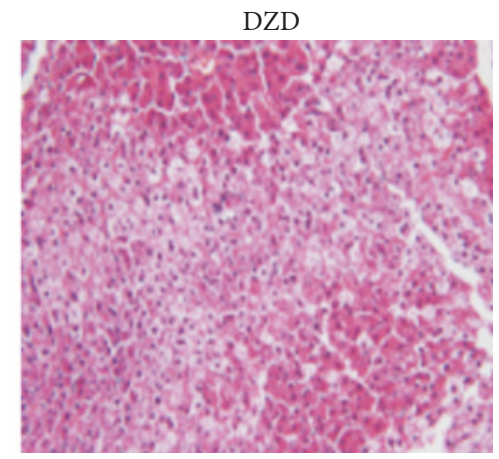

FIGURE 1: Therapeutic effects of DZD on rat model of HFD-induced NAFLD. Male SD rats (8 weeks old) were fed HFD for 16 weeks, followed by either 4-week DZD feeding or normal saline supplementation through gavage, while the rats fed normal diet were set as controls. The rats were sacrificed, from which the liver and serum were collected. Body weight (a), liver weight (b), serum ALT (c), serum AST (d), serum TG (e), serum TC (f), serum HDL-C (g), serum LDL-C (h), and serum GLU (i) levels were detected. Liver sections were stained with HE (j). The data are expressed as mean \pm SEM. $N=5$ to 8 for every group. ${ }^{*} 0.01<P<0.05 ;{ }^{* *} P<0.01$. ALT: alanine aminotransferase; AST: aspartate transaminase; TG: triglyceride; TC: total cholesterol; HDL-C: high density lipoprotein-cholesterol; LDL-C: low density lipoproteincholesterol; GLU: glucose; HE: hematoxylin/eosin. 


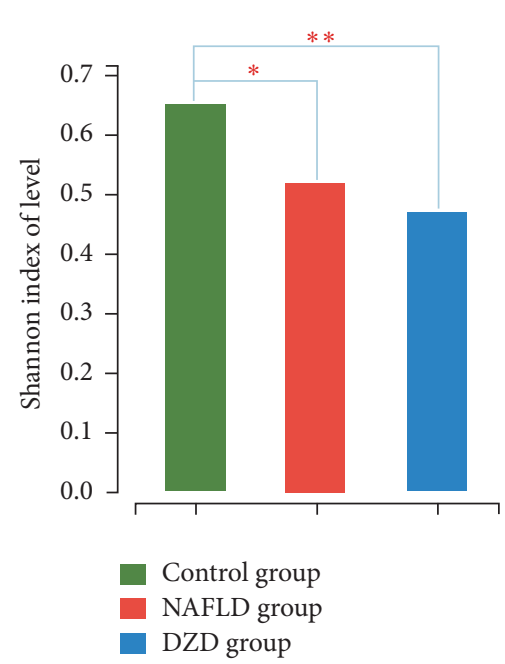

(a)

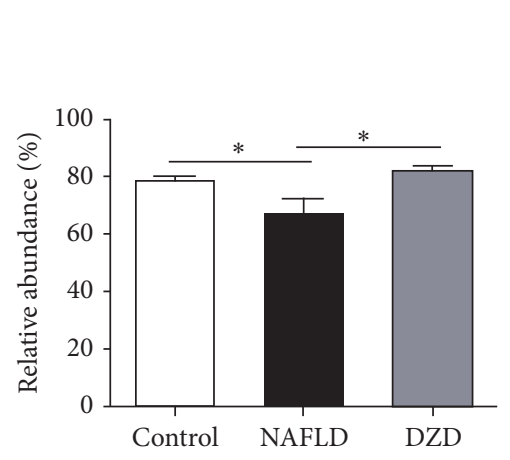

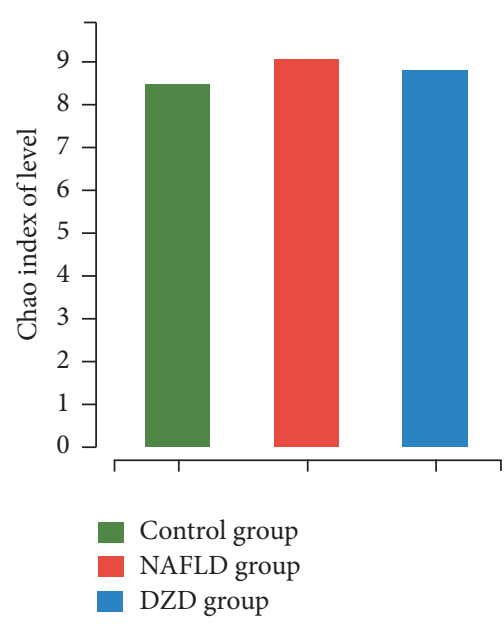

(b)

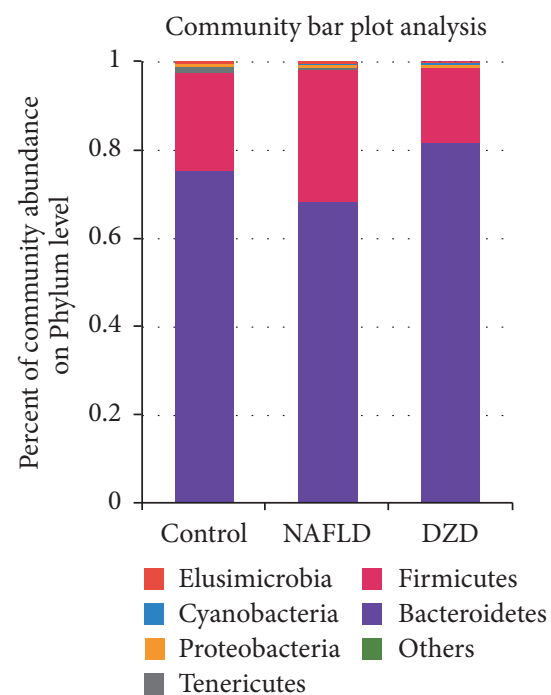

(c) (d)

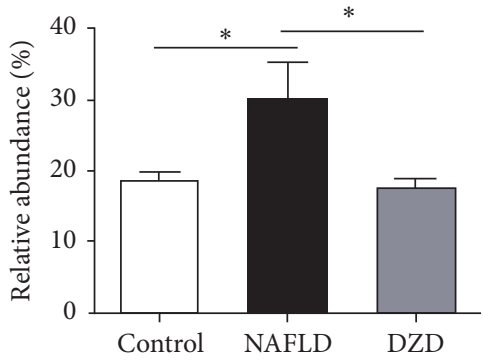

(e)

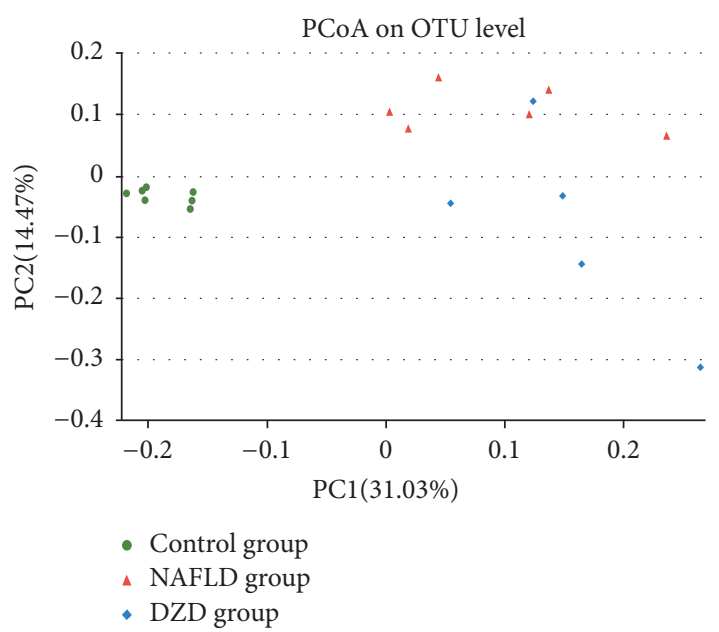

(f)

Figure 2: Effects of DZD therapy on composition of gut microbiota. Bacterial diversity is shown by the Shannon index (a). Bacterial richness is shown by the Chao index (b). Species on the phylum level (c), as well as alterations of Bacteroidetes (d) and Firmicutes (e) are indicated. PCoA score plot based on the unweighted significance test (f). The data are expressed as mean \pm SEM. $N=5$ to 8 for every group. ${ }^{*} 0.01<P<0.05$; ${ }^{* *} P<0.01$.

group, which were reversed by DZD intervention (Figures 3(c) and 3(d)). Meanwhile, the relative abundances of Bacteroides, Oscillibacter, and Butyricicoccus were significantly lower in the NAFLD group, which were restored by DZD intervention (Figures 3(e)-3(g)). In short, DZD altered the intestinal microbiota composition of NAFLD rats.

(3) DZD Intervention Activated the TLR4 Signaling Pathway in the Ileum. Damage of the intestinal barrier may induce intestinal endotoxemia and subsequent TLR4 activation in the liver, which is implicated in the pathogenesis of NAFLD $[15,16]$. Kim et al. reported that HFD-induced intestinal inflammatory response by activating the TLR4 signaling pathway [17]. To further investigate whether DZD regulated intestinal inflammation via this pathway, the expressions of TLR4 and mitogen-activated protein kinase (MAPK) members in the ileum were detected by Western blotting. The protein expression levels of TLR4 and MyD88 were upregulated in the ileum of the HFD group (Figures 4(a)-4(c)). After treatment with DZD, such levels were restored to normal (Figures 4(a)-4(c)). The phosphorylation levels of ERK and JNK in the HFD group exceeded those of the control group (Figures 4(d), 4(e), and 4(g)). However, the three groups had similar ERK and JNK levels (Figures 4(d), 4(f), and 4(h)). Similarly, DZD downregulated the phosphorylation levels of these proteins (Figures 4(d), 4(e), and 4(g)). Thus, activation of the TLR4 signaling pathway in the ileum was enhanced by HFD, whereas DZD reduced the activity of this pathway to normal.

(4) DZD Intervention Activated the TLR4 Signaling Pathway in the Colon. We also detected the protein expression levels of 


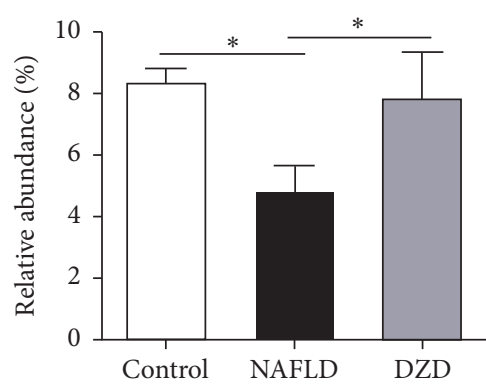

(a)

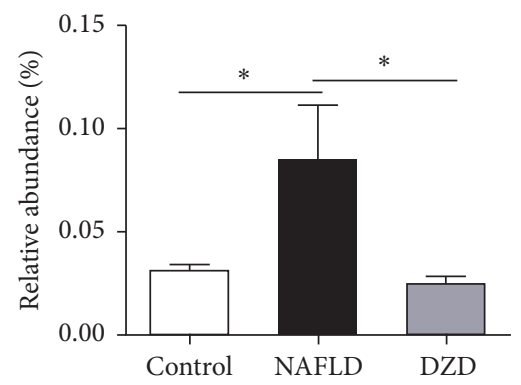

(d)

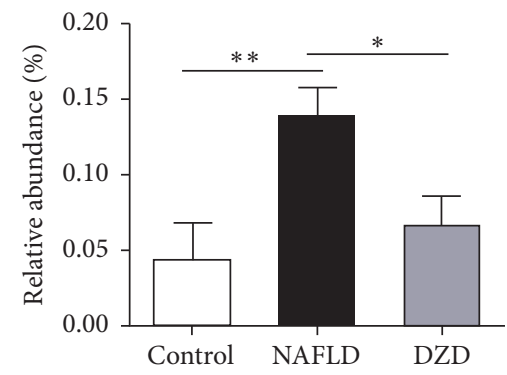

(b)

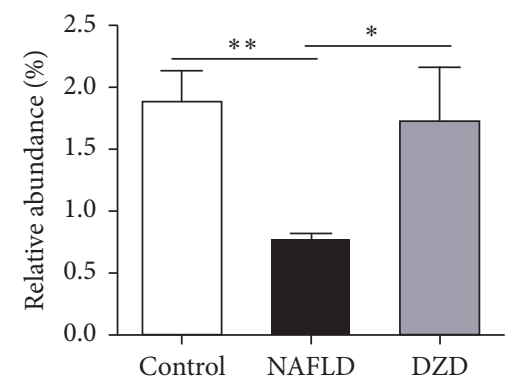

(e)

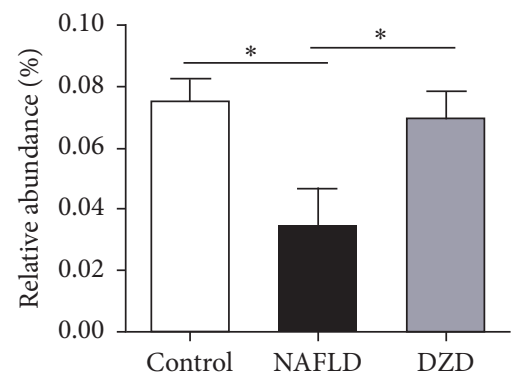

(g)

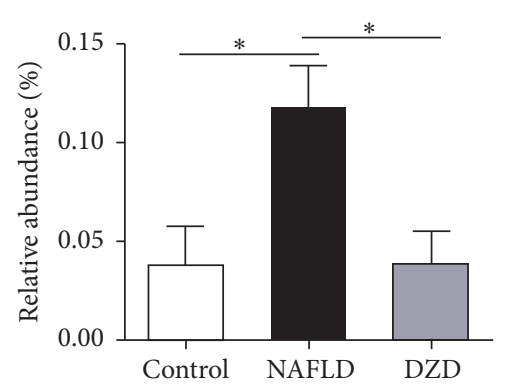

(c)

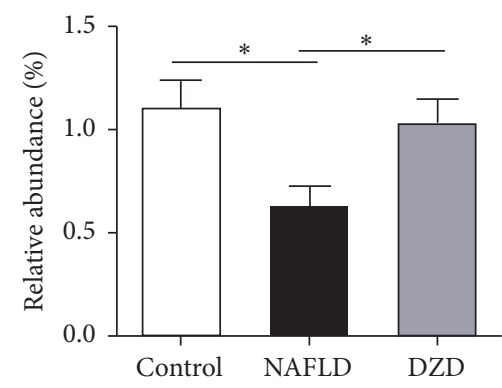

(f)

FIGURE 3: Relative abundances of gut microbiota on different taxonomic levels among three groups. On the family level, the alterations of Ruminococcaceae (a) and Desulfovibrionaceae (b) were indicated. At the genus level, the alterations of Desulfovibrio (c), Escherichia/Shigella (d), Bacteroides (e), Oscillibacter (f), and Butyricicoccus (g) were indicated. The data are expressed as mean \pm SEM. $N=5$ to 8 for every group. ${ }^{*} 0.01<P<0.05$; ${ }^{* *} P<0.01$.

members in the TLR4 signaling pathway in the colon of different groups. HFD upregulated the expression levels of TLR4 and MyD88 in the colon (Figures 5(a)-5(c)). Compared to the HFD group, DZD intervention downregulated their protein expressions (Figures 5(a)-5(c)). Nevertheless, there was no significant difference between control and DZD groups. Identical to results in the ileum, the phosphorylation levels of ERK and JNK in the HFD group were higher than those in the control group, which were downregulated by DZD (Figures 5(d), 5(e), and 5(g)). Similarly, the total protein expression levels of ERK and JNK in the colon were not significantly different among the three groups (Figures 5(d), 5(f), and 5(h)). Taken together, HDF caused activation of the TLR4 signaling pathway in the colon, which was attenuated by DZD.

(5) DZD Intervention Relieved Loss of Intestinal Barrier Integrity in the NAFLD Model. TJ proteins of the intestinal mucosa, such as ZO-1 and occludin, are crucial to maintenance of the intestinal barrier [18]. Decrease in the expressions of ZO-1 and occludin can increase the intestinal permeability and play an important role in the pathophysiology of NAFLD [19]. To assess the effects of HFD and DZD on the intestinal barrier function, we used immunohistochemistry to detect the protein expression levels of $\mathrm{ZO}-1$ and occludin in the gut. In accordance with previous studies, ZO-1 and occludin were highly expressed in both the ileum and colon of the control group, which were reduced by HFD intervention (Figures 6(a)-6(f)). After treatment with DZD, the protein expressions of ZO-1 and occludin in the gut were recovered compared with those of the HFD group (Figures 6(a)-6(f)). Therefore, the expressions of ZO-1 and occludin in the gut, which were reduced by HDF, were restored by DZD, thereby mitigating the loss of intestinal barrier integrity in the NAFLD model.

(6) Identification of Components of DZD. The components of DZD water extract were identified by HPLC-MS. Ten 


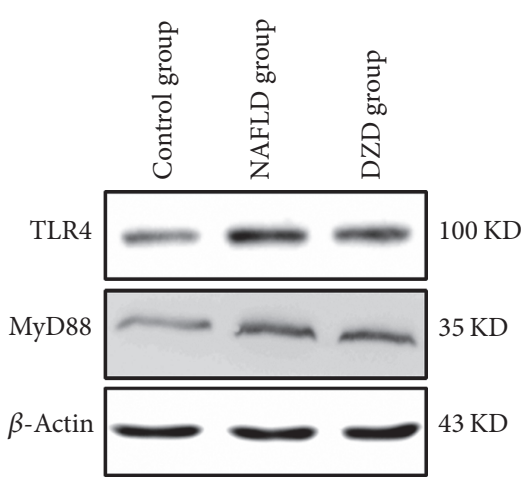

(a)

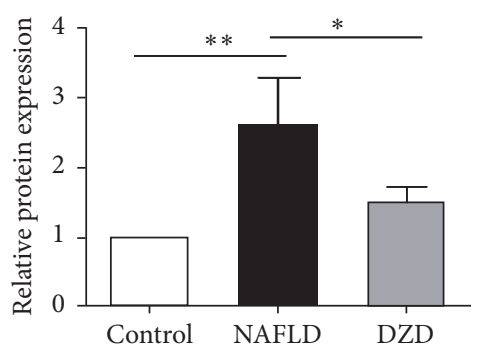

(b)

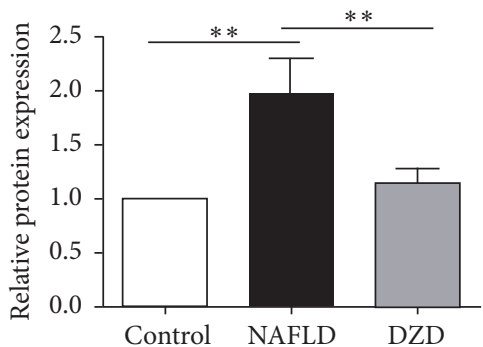

(c)

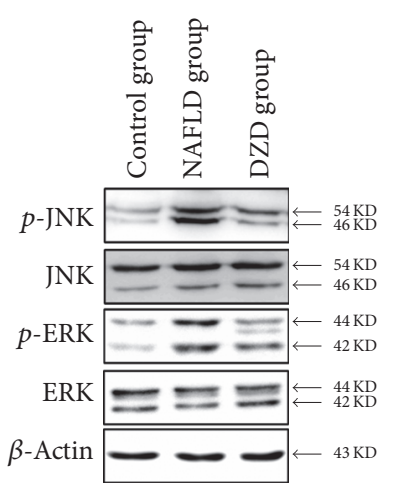

(d)

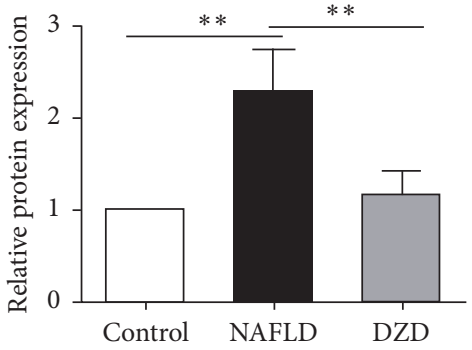

(e)

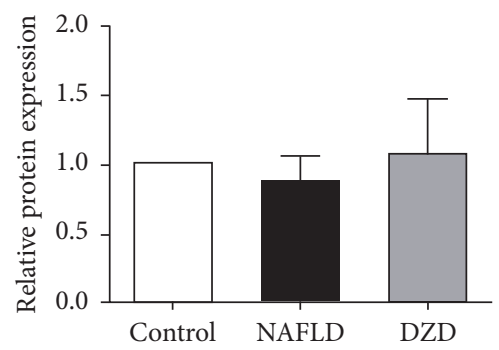

(f)

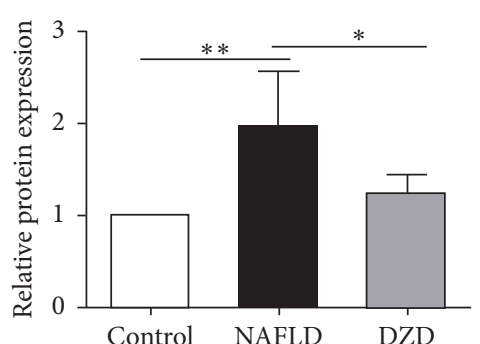

(g)

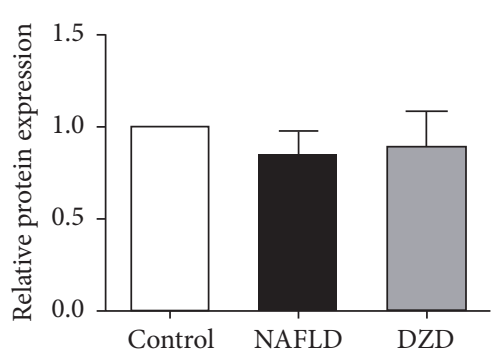

(h)

FIGURE 4: DZD intervention activated the TLR4 signaling pathway of the ileum. The ilea of rats were collected. TLR4, MyD88 (a), p-JNK, JNK, p-ERK, and ERK (d) protein expressions of ileum tissues were analyzed by Western blot. Bar graphs are the plots of TLR4 (b), MyD88 (c), $p$-JNK (e), JNK (f), $p$-ERK (g), and ERK (h). The data are expressed as mean \pm SEM. $N=5$ to 8 for every group. ${ }^{*} 0.01<P<0.05$; ${ }^{* *} P<0.01$.

potential compounds, that is, gallic acid, chrysophanol, rhein, emodin, physcion, alisol C monoacetate, alisol B, atractylenolide I, atractylenolide II, and atractylenolide III, were identified (Figure 7, Table 1). The characterizations and sources of these compounds are listed in Table 1.

\section{Discussion}

NAFLD is the liver manifestation of metabolic syndrome, with a high incidence rate worldwide $[20,21]$. At present, NAFLD has become the main cause for hepatocellular carcinoma in the United States $[22,23]$. There are still no effective therapies for NAFLD patients in Western countries [24]. Contrarily, the therapeutic effects of traditional Chinese medical formulations on NAFLD have been well-documented.
Our study firstly demonstrated that DZD mitigated HFDinduced NAFLD. Sixteen weeks of HFD feeding increased body weight, as well as disturbing liver function parameters and blood lipid levels (Figures 1(a)-1(h)). Liver histological examination revealed extensive macrosteatosis and hepatocyte ballooning (Figure $1(\mathrm{j})$ ), being consistent with previous studies. We also demonstrated that DZD administration for 4 weeks reduced body weight and blood lipid levels, improved liver function parameters, and alleviated the liver pathological changes of NAFLD rats (Figures $1(\mathrm{a})-1(\mathrm{~h})$ and $1(j))$. Hence, DZD indeed relieved HFD-induced NAFLD.

In sequencing studies, the microbiota composition of NAFLD patients markedly changed after HFD feeding, so DZD may mitigate NAFLD by altering the gut microbiota following oral administration. Firstly, we assessed the effect of DZD on the gut microbiota by using multivariate analysis. 
TABLE 1: Identification of potential components of DZD.

\begin{tabular}{|c|c|c|c|c|c|}
\hline Number & Rt (min) & Molecular formula & Molecular weight $(\mathrm{m} / z)$ & Observed ion peaks $(\mathrm{m} / z)$ & Identification \\
\hline (1) & 1.05 & $\mathrm{C} 7 \mathrm{H} 6 \mathrm{O} 5$ & 170.12 & $169[\mathrm{M}-\mathrm{H}]^{-}, 125$ & Gallic acid \\
\hline (2) & 13.56 & $\mathrm{C} 15 \mathrm{H} 10 \mathrm{O} 4$ & 254.23 & $254[\mathrm{M}+\mathrm{H}]^{+}, 237$ & Chrysophanol \\
\hline (3) & 14.08 & $\mathrm{C} 15 \mathrm{H} 8 \mathrm{O} 6$ & 284.22 & $283[\mathrm{M}-\mathrm{H}]^{-}, 239$ & Rhein \\
\hline (4) & 16.67 & $\mathrm{C} 15 \mathrm{H} 10 \mathrm{O} 5$ & 270.24 & $269[\mathrm{M}-\mathrm{H}]^{-}, 240.9,225,180.9$ & Emodin \\
\hline (5) & 14.31 & $\mathrm{C} 16 \mathrm{H} 12 \mathrm{O} 5$ & 284.27 & $283[\mathrm{M}-\mathrm{H}]^{-}, 268$ & Physcion \\
\hline (6) & 14.62 & $\mathrm{C} 32 \mathrm{H} 48 \mathrm{O} 6$ & 528.728 & $529[\mathrm{M}+\mathrm{H}]^{+}, 511,469,451,415$ & Alisol C monoacetate \\
\hline (7) & 18.31 & $\mathrm{C} 30 \mathrm{H} 48 \mathrm{O} 4$ & 472.70 & $473[\mathrm{M}+\mathrm{H}]^{+}, 455,437,383,365$ & Alisol B \\
\hline (8) & 15.46 & $\mathrm{C} 15 \mathrm{H} 18 \mathrm{O} 2$ & 230.30 & $231[\mathrm{M}+\mathrm{H}]^{+}, 213,203,185,157,143$ & Atractylenolide I \\
\hline (9) & 17.79 & $\mathrm{C} 15 \mathrm{H} 20 \mathrm{O} 2$ & 232.32 & $233[\mathrm{M}+\mathrm{H}]^{+}, 215,197,187,159,151$ & Atractylenolide II \\
\hline$(10)$ & 15.47 & $\mathrm{C} 15 \mathrm{H} 20 \mathrm{O} 3$ & 248.32 & $249[\mathrm{M}+\mathrm{H}]^{+}, 231,203,189,163,135.69$ & Atractylenolide III \\
\hline
\end{tabular}

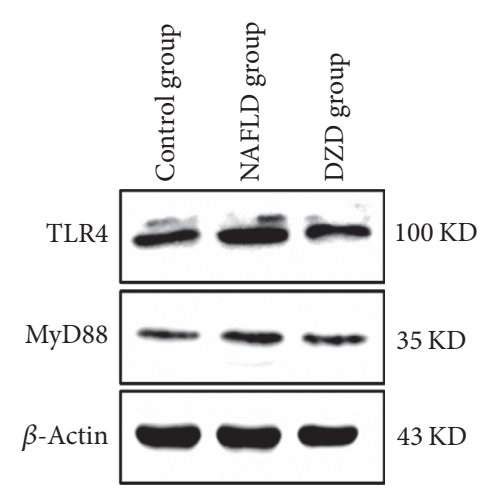

(a)

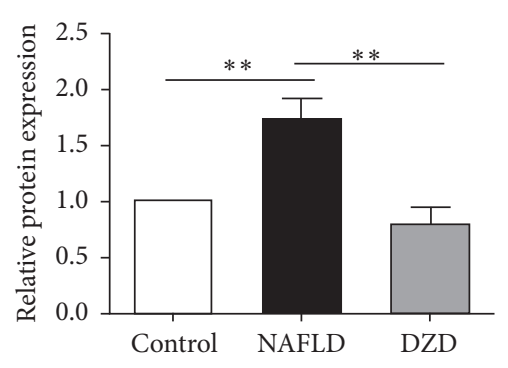

(b)

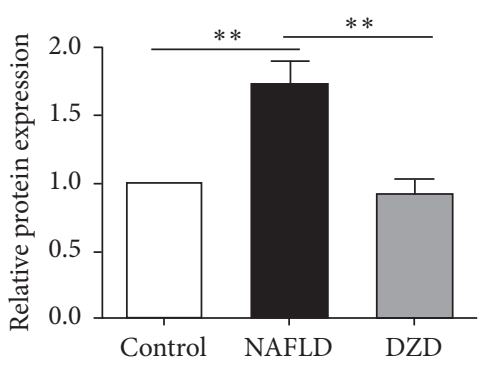

(c)

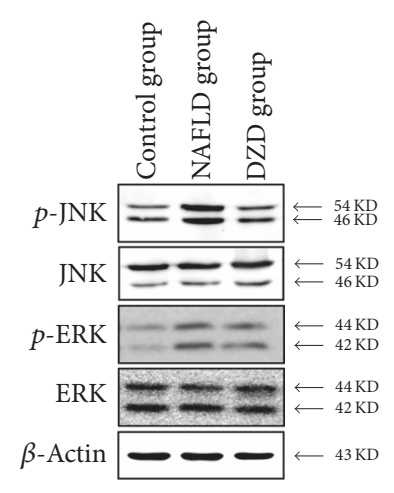

(d)

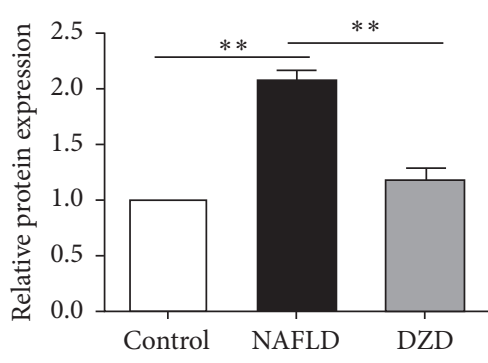

(e)

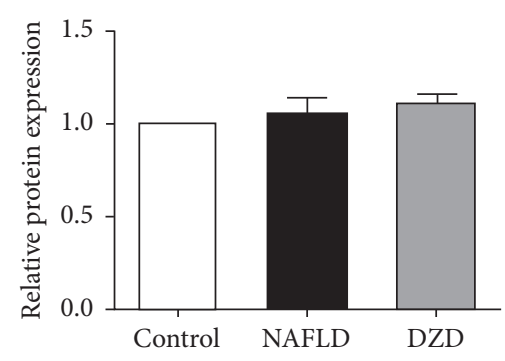

(f)

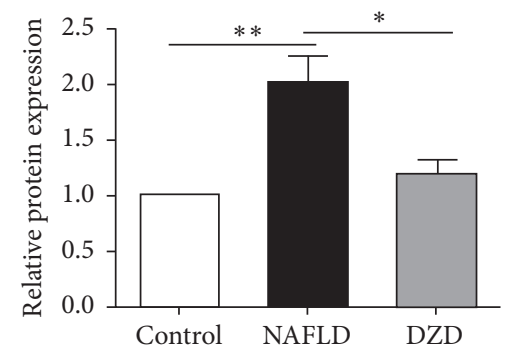

(g)

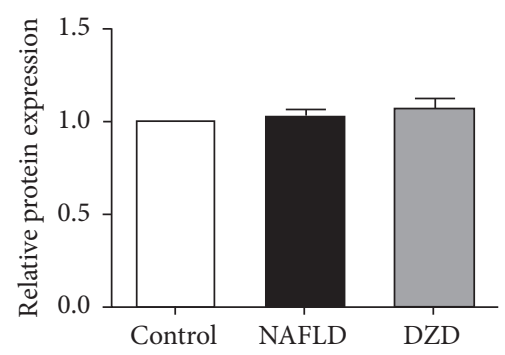

(h)

FIGURE 5: DZD intervention activated the TLR4 signaling pathway of the colon. The colons of rats were collected. TLR4, MyD88 (a), p-JNK, JNK, $p$-ERK, and ERK (d) protein expressions of colon tissues were analyzed by Western blot. Bar graphs are the plots of TLR4 (b), MyD88 (c), $p$-JNK (e), JNK (f), $p$-ERK (g), and ERK (h). The data are expressed as mean \pm SEM. $N=5$ to 8 for every group. ${ }^{*} 0.01<P<0.05$; ${ }^{* *} P<0.01$. 


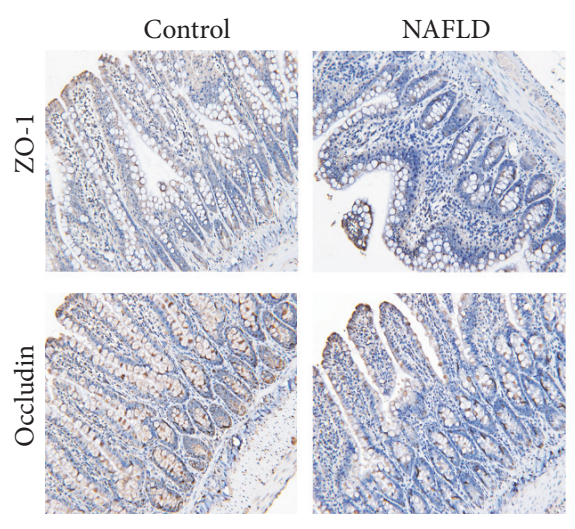

(a)

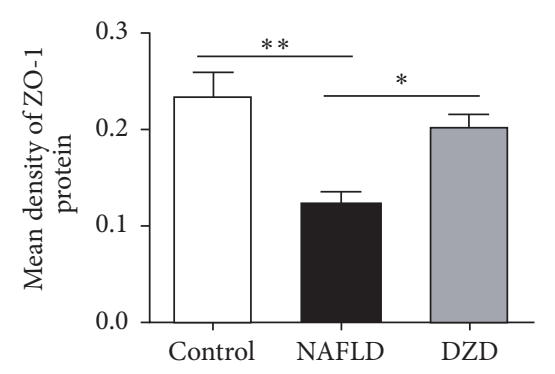

(c)
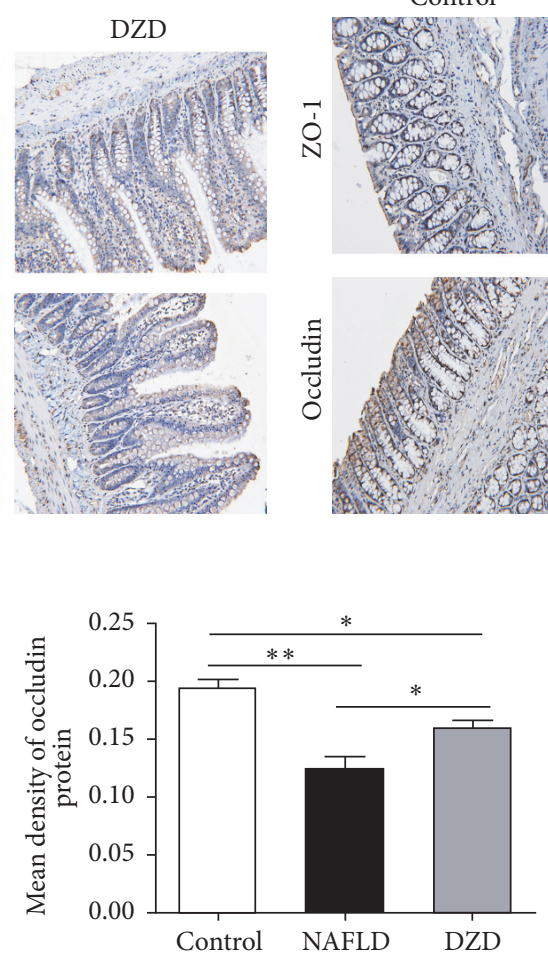

(d)

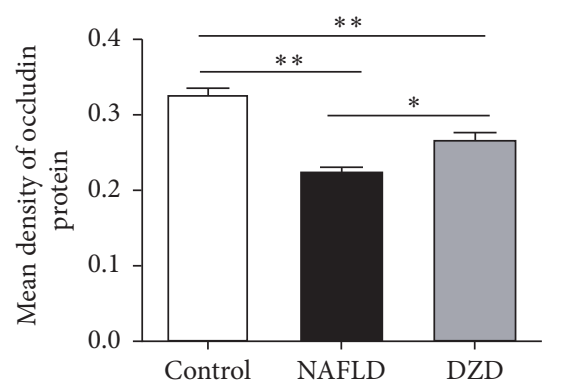

(f)
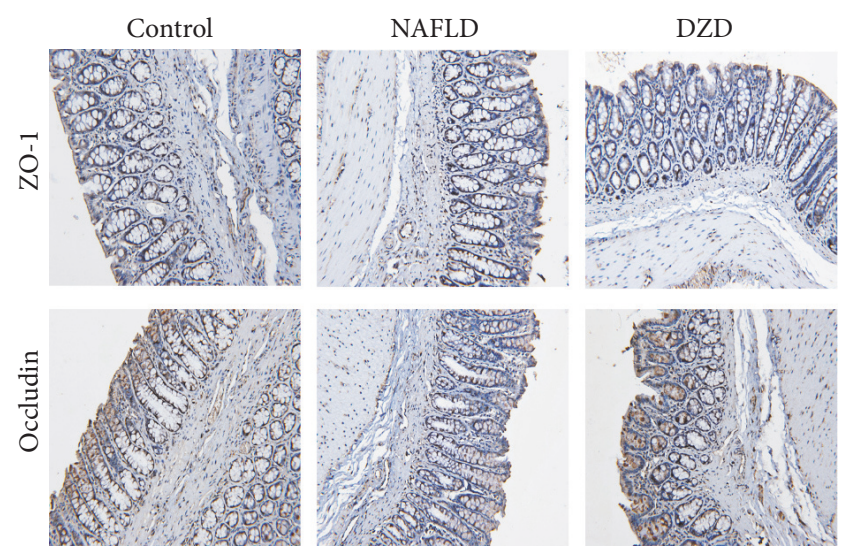

(b)

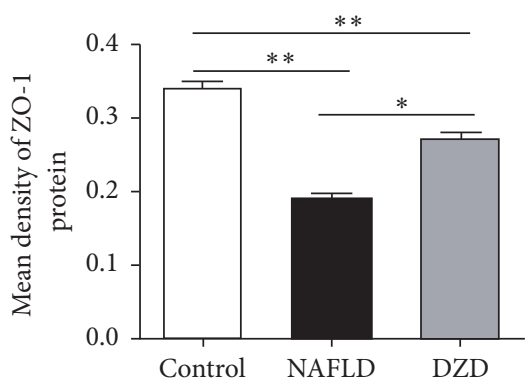

(e)

FIGURE 6: DZD intervention alleviated loss of intestinal TJ proteins. Immunohistochemical staining for ZO-1 and occludin in ileum (a) and colon (b) tissues. Bar graphs are the plots of ZO-1 (c) and occludin (d) in the ileum and ZO-1 (e) and occludin (f) in the colon. The data are expressed as mean \pm SEM. $N=5$ to 8 for every group. ${ }^{*} 0.01<P<0.05 ;{ }^{* *} P<0.01$.

The Shannon index and Chao index represented the diversity and richness of gut microbiota in each sample, respectively. HFD and DZD both significantly reduced the diversity of gut microbiota compared with that of the control group (Figure 2(a)). However, the three groups had similar richness (Figure 2(b)). Subsequently, we detected the gut microbiotas in different groups on the phylum level. The NAFLD group had lower percentage of Bacteroidetes but higher percentage of Firmicutes (Figures 2(c)-2(e)), similar to previous studies [25-27]. Nevertheless, the results were restored by DZD treatment (Figures 2(c)-2(e)).

The serum levels of LPS, as a gut bacteria-derived product, are elevated in NAFLD patients. LPS is an important component of the outer membrane of Gram-negative bacteria of which Bacteroidetes is the main type. In our study, Bacteroidetes decreased in the NAFLD group, but damage of the intestinal barrier and increase of the gut permeability induced more gut-derived bacterial LPS to enter blood via the hepatic portal system [28]. The integrity of the intestinal barrier is closely related to gut bacteria-derived products, and LPS can increase the gut permeability by inducing intestinal inflammatory response. Therefore, we further tested the relative abundances of Gram-negative bacteria on family and genus levels. Bacteroides, a genus of Bacteroidetes, changed following the same trend. Interestingly, the fecal microbiome of the NAFLD group had increased Desulfovibrionaceae family, Desulfovibrio genus, and Escherichia/Shigella genus (Figures 3(b)-3(d)). The relative abundances of Desulfovibrionaceae, a family of sulfate-reducing bacteria (SRB), and Desulfovibrio, a genus of SRB, increased in the NAFLD group, which were reversed by DZD (Figures 3(b) and 3(c)). A previous study showed that diet increased SRB in vitro [29]. These gut 


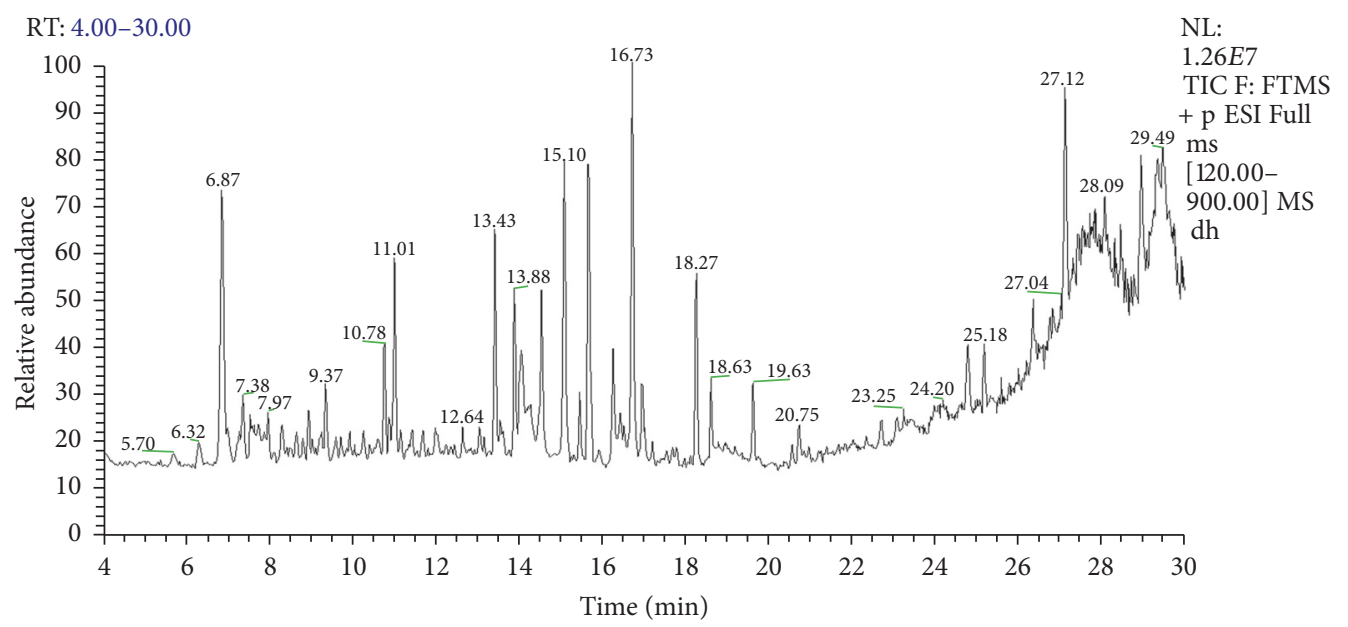

(a)

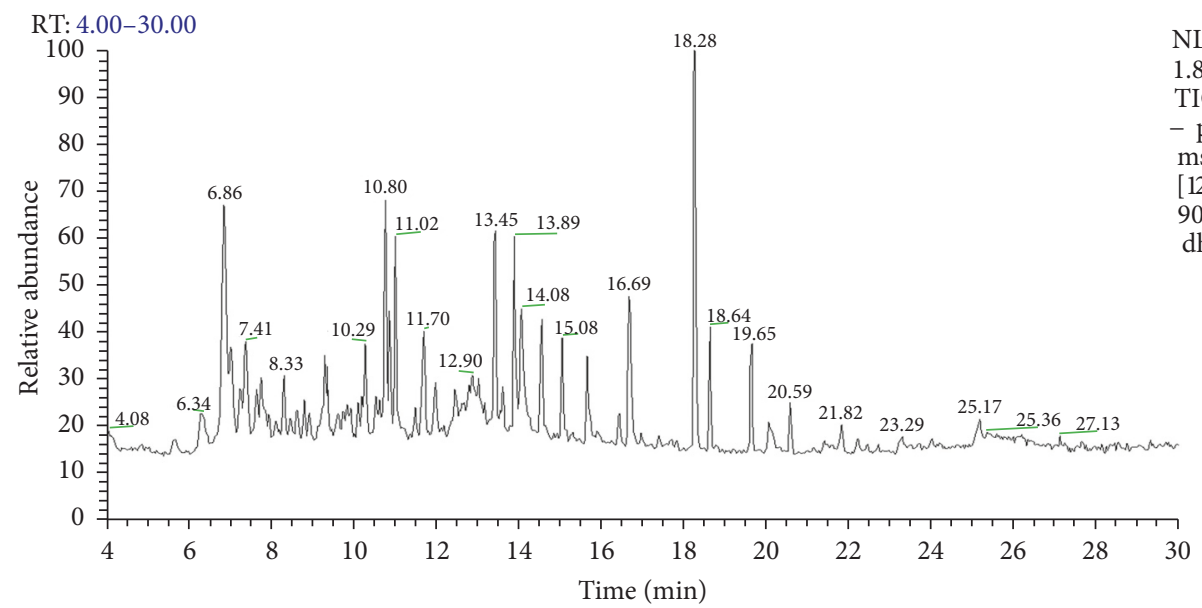

NL:

$84 \mathrm{E} 7$

TIC F: FTMS

- p ESI Full

$[120.00$

900.00] MS

dh-neg

(b)

Figure 7: HPLC chromatogram of DZD. Total ions chromatograms of DZD in positive (a) and negative (b) ion modes. Identification of potential constituents of DZD (see Table 1).

bacteria can release hydrogen sulfide which is a genotoxic gas that affects epithelial intestinal cell integrity and causes barrier dysfunction [30]. Meanwhile, Lodowska et al. verified the high biological activity of LPS from Desulfovibrio [31]. Although these bacteria have low contents, LPS in them can evidently induce inflammation. Herein, Escherichia/Shigella genus was significantly enriched in the NAFLD group compared to that in the control group, which decreased to normal levels after DZD treatment (Figure 3(d)). Increase of Escherichia/Shigella can induce intestinal inflammation, causing intestinal barrier dysfunction [32, 33]. Therefore, even a small number of Gram-negative bacteria can still destroy the intestinal barrier through intense proinflammatory effects.

In our study, the fecal microbiome of the NAFLD group had decreased Ruminococcaceae family, Oscillibacter genus, and Butyricicoccus genus (Figures 3(a), 3(f), and 3(g)), but the results were restored by DZD treatment. Jiang et al. demonstrated that the Ruminococcaceae family and the Oscillibacter genus increased significantly in the healthy group compared to those in the NAFLD group [34]. Ruminococcaceae, Oscillibacter, and Butyricicoccus are short chain fatty acid- (SCFA) producing bacteria which prevent metabolic endotoxemia by strengthening the gut barrier [35-37]. SCFAs can protect against gut inflammation and decrease intestinal permeability $[38,39]$. Changes of these gut bacteria induce decrease of SCFAs, increasing the intestinal permeability. Nevertheless, we herein proved that DZD invention restored the changes of these gut bacteria induced by HFD.

In this study, we focused on the influence of gut microbiota-mediated inflammation activation on the intestinal barrier, so we detected the changes of inflammatory signaling pathway in the gut. LPS is a special pathogen-associated molecular pattern and one of microbial products, being able to activate inflammatory pathways by binding TLR4. As a result, intestinal inflammatory response then occurs [40]. Studies have suggested that TLR4-mediated signaling of the gut potently drove the progression of NAFLD [41, 42]. As the receptor of LPS, TLR4 is expressed on the membranes of hepatocytes, IECs, immune cells, and so on. MyD88 is a 
downstream adaptor protein for all TLRs, except for TLR3 [43]. The TLR4-MyD88-MAPKs signaling cascade is crucial to inflammatory response and NAFLD progression. The MAPK signaling pathway is involved in a variety of physiological and pathological processes such as cell growth, inflammation, apoptosis, and proliferation [44-46]. ERK MAPK and JNK MAPK are two main components of the MAPK pathway. Activating TLR4 in the gut induces the inflammation of intestinal mucosa by mediating the phosphorylation of ERK and JNK. To further investigate whether DZD can regulate intestinal inflammation via the TLR4 signaling pathway, the expressions of members in the TLR4 signaling pathway of the ileum and colon were detected by Western blotting. The protein expression levels of TLR4 and MyD88 in the ileum were upregulated in the HFD group (Figures 4(a)-4(c)). After treatment with DZD, such levels were restored to normal (Figures 4(a)-4(c)). The total protein levels of ERK and JNK were similar among the three groups (Figures 4(d), 4(f), and 4(h)). However, the phosphorylation levels of these two proteins in the HFD group surpassed those of the control group (Figures 4(d), 4(e), and 4(g)). DZD downregulated the phosphorylation levels of ERK and JNK in NAFLD rats (Figures 4(d), 4(e), and 4(g)). These results confirmed that activation of the TLR4 signaling pathway in the ileum was upregulated by HFD. Meanwhile, DZD recovered the activity of the TLR4 signaling pathway to normal. Similar results were observed in the colon (Figures $5(\mathrm{a})-5(\mathrm{~h})$ ). Taken together, activation of TLR4 signaling in both the ileum and colon, which was induced by HDF, was downregulated by DZD.

Microbiota participates in liver diseases largely via the inflammatory pathway triggered by the interactions between intestinal bacteria and the intestinal barrier. The increased intestinal permeability in NAFLD patients has been attributed to intestinal microbiota [47]. Additionally, the gut microbiota influences the intestinal barrier function, while dysfunction of this barrier and LPS is closely related in human and animal models [48, 49]. A former research reported that LPS significantly induced the downregulation and redistribution of TJ proteins in $\mathrm{Caco} 2$ monolayers, as well as promoting the increase of intestinal epithelial permeability [50]. Damage of the intestinal barrier may result in translocation of intestinal bacteria and then entrance of gutderived pathogens into portal circulation through the highly permeable intestinal barrier, triggering NAFLD eventually [51-53]. TJ proteins suppress the paracellular permeability and thus contribute essentially to the intestinal barrier, as a defense line, thereby impeding the entrance of intestinal pathogens into the liver. Intestinal permeability is regulated by TJ proteins of which transmembrane proteins $\mathrm{ZO}-1$ and occludin have attracted wide attention. Given that ZO-1 and occludin are of great significance to the integrity of TJs, their downregulation is responsible for the disruption of TJ structure and the increase in paracellular permeability [54, 55]. In this study, immunohistochemistry revealed that HFD deceased the expressions of ZO-1 and occludin in both the ileum and colon (Figures 6(a)-6(f)). Accordingly, DZD upregulated such expressions (Figures 6(a)-6(f)) and then boosted the intestinal barrier function.
Furthermore, we analyzed the main components of DZD by using high-performance liquid chromatography coupled with mass spectrometry and successfully identified ten potential components of DZD, that is, gallic acid, chrysophanol, rhein, emodin, physcion, alisol C monoacetate, alisol $\mathrm{B}$, atractylenolide I, atractylenolide II, and atractylenolide III (Figure 7, Table 1). Huang et al. demonstrated that gallic acid could improve high-fat diet- (HFD-) induced dyslipidaemia and hepatosteatosis [56]. Meng et al. reported that alisol $\mathrm{B}$ protected against nonalcoholic steatohepatitis in mice by activating farnesoid $X$ receptor [57]. Rhein and emodin also can improve NAFLD induced by HFD $[58,59]$, and emodin can restore the increased intestinal permeability by inhibiting inflammation response [60]. Meanwhile, a former research revealed that alisol $\mathrm{C}$ monoacetate and alisol B could inhibit LPS-induced inflammatory reaction [61]. Although it is unclear whether atractylenolide can improve NAFLD, it has a definite anti-inflammatory and antiapoptosis effect $[62,63]$. Due to complicated components of Chinese herbal medicine, we only identified ten of the main components of DZD. Further studies are still needed to clarify the main components of DZD and the potential mechanism for the therapeutic effects of DZD on NAFLD.

In conclusion, intestinal dysbacteriosis, activation of the TLR4 signaling pathway in the gut, and intestinal barrier dysfunction played important roles in NAFLD. Moreover, DZD changed intestinal bacterial communities, inhibited the intestinal TLR4 signaling pathway, restored the expressions of TJ proteins in the gut, and finally relieved HFD-induced NAFLD. Gallic acid, chrysophanol, rhein, emodin, physcion, alisol C monoacetate, alisol B, atractylenolide I, atractylenolide II, and atractylenolide III were ten main components of DZD. However, further studies are still needed to unravel the mechanism by which DZD treats NAFLD.

\section{Conflicts of Interest}

The authors declare that there are no conflicts of interest regarding the publication of this paper.

\section{Acknowledgments}

(1) This work was supported by grants from the National Natural Science Foundation of China (no. 81403376). (2) The authors gratefully acknowledge the valuable cooperation of Dr. Wen-bing Shang and the members of his laboratory in preparing this study.

\section{References}

[1] P. Almeda-Valdés, D. Cuevas-Ramos, and C. A. Aguilar-Salinas, "Metabolic syndrome and non-alcoholic fatty liver disease," Annals of Hepatology, vol. 8, no. 1, pp. S18-S24, 2009.

[2] J. C. Cohen, J. D. Horton, and H. H. Hobbs, "Human fatty liver disease: old questions and new insights," Science, vol. 332, no. 6037, pp. 1519-1523, 2011.

[3] S. Townsend and P. N. Newsome, "Non-alcoholic fatty liver disease in 2016," British Medical Bulletin, vol. 119, no. 1, pp. 143156, 2016. 
[4] S. Milić and D. Štimac, "Nonalcoholic fatty liver disease/steatohepatitis: epidemiology, pathogenesis, clinical presentation and treatment," Digestive Diseases, vol. 30, no. 2, pp. 158-162, 2012.

[5] C. D. Williams, J. Stengel, M. I. Asike et al., "Prevalence of nonalcoholic fatty liver disease and nonalcoholic steatohepatitis among a largely middle-aged population utilizing ultrasound and liver biopsy: a prospective study," Gastroenterology, vol. 140, no. 1, pp. 124-131, 2011.

[6] Z. M. Younossi, A. B. Koenig, D. Abdelatif, Y. Fazel, L. Henry, and M. Wymer, "Global epidemiology of nonalcoholic fatty liver disease-meta-analytic assessment of prevalence, incidence, and outcomes," Hepatology, vol. 64, no. 1, pp. 73-84, 2016.

[7] A. Wieland, D. N. Frank, B. Harnke, and K. Bambha, "Systematic review: microbial dysbiosis and nonalcoholic fatty liver disease," Alimentary Pharmacology \& Therapeutics, vol. 42, no. 9, pp. 1051-1063, 2015.

[8] V. Tremaroli and F. Bäckhed, "Functional interactions between the gut microbiota and host metabolism," Nature, vol. 489, no. 7415, pp. 242-249, 2012.

[9] S. Guercio Nuzio, M. Di Stasi, L. Pierri et al., "Multiple gut-liver axis abnormalities in children with obesity with and without hepatic involvement," Pediatric Obesity, vol. 28, no. 6, pp. 1-7, 2016.

[10] G. Paolella and P. Vajro, "Childhood obesity, breastfeeding, intestinal microbiota, and early exposure to antibiotics: what is the link?” JAMA Pediatrics, vol. 170, no. 8, pp. 735-737, 2016.

[11] M. Mouzaki, E. M. Comelli, B. M. Arendt et al., "Intestinal microbiota in patients with nonalcoholic fatty liver disease," Hepatology, vol. 58, no. 1, pp. 120-127, 2013.

[12] M. Raman, I. Ahmed, P. M. Gillevet et al., "Fecal microbiome and volatile organic compound metabolome in obese humans with nonalcoholic fatty liver disease," Clinical Gastroenterology and Hepatology, vol. 11, no. 7, pp. 868-875.e3, 2013.

[13] A. M. Marchiando, W. V. Graham, and J. R. Turner, "Epithelial barriers in homeostasis and disease," Annual Review of Pathology: Mechanisms of Disease, vol. 5, pp. 119-144, 2010.

[14] G. Zhu, G. Zhang, and M. Wang, "Study on Hypolipidemic Effect of Zexie Decoction with Different Compatibility Proportions," Chinese Archives of Traditional Chinese Medicine, vol. 33, no. 1, pp. 189-191, 2015.

[15] L. Xue, J. He, N. Gao et al., "Probiotics may delay the progression of nonalcoholic fatty liver disease by restoring the gut microbiota structure and improving intestinal endotoxemia," Scientific Reports, vol. 7, Article ID 45176, 2017.

[16] L. Miele, V. Valenza, G. La Torre et al., "Increased intestinal permeability and tight junction alterations in nonalcoholic fatty liver disease," Hepatology, vol. 49, no. 6, pp. 1877-1887, 2009.

[17] K.-A. Kim, I.-A. Lee, W. Gu, S. R. Hyam, and D.-H. Kim, “ $\beta$ Sitosterol attenuates high-fat diet-induced intestinal inflammation in mice by inhibiting the binding of lipopolysaccharide to toll-like receptor 4 in the NF- $\kappa$ B pathway," Molecular Nutrition \& Food Research, vol. 58, no. 5, pp. 963-972, 2014.

[18] T. Suzuki, "Regulation of intestinal epithelial permeability by tight junctions," Cellular and Molecular Life Sciences, vol. 70, no. 4, pp. 631-659, 2013.

[19] A. R. Moschen, S. Kaser, and H. Tilg, "Non-alcoholic steatohepatitis: a microbiota-driven disease," Trends in Endocrinology \& Metabolism, vol. 24, no. 11, pp. 537-545, 2013.

[20] N. Chalasani, Z. Younossi, and J. E. Lavine, "The diagnosis and management of non-alcoholic fatty liver disease: practice guideline by the American Association for the Study of Liver
Diseases, American College of Gastroenterology, and the American Gastroenterological Association," Hepatology, vol. 55, no. 6, pp. 2005-2023, 2012.

[21] G. Vernon, A. Baranova, and Z. M. Younossi, "Systematic review: the epidemiology and natural history of non-alcoholic fatty liver disease and non-alcoholic steatohepatitis in adults," Alimentary Pharmacology \& Therapeutics, vol. 34, no. 3, pp. 274-285, 2011.

[22] S. Mittal and H. B. El-Serag, "Epidemiology of hepatocellular carcinoma: consider the population," Journal of Clinical Gastroenterology, vol. 47, no. 1, pp. S2-S6, 2013.

[23] S. Mittal, H. B. El-Serag, Y. H. Sada et al., "Hepatocellular carcinoma in the absence of cirrhosis in united states veterans is associated with nonalcoholic fatty liver disease," Clinical Gastroenterology and Hepatology, vol. 14, no. 1, pp. 124-131.el, 2016.

[24] T. Charytoniuk, K. Drygalski, K. Konstantynowicz-Nowicka, K. Berk, and A. Chabowski, "Alternative treatment methods attenuate the development of NAFLD: a review of resveratrol molecular mechanisms and clinical trials," Nutrition Journal, vol. 34, pp. 108-117, 2017.

[25] Q. Feng, W. Liu, S. S. Baker et al., "Multi-targeting therapeutic mechanisms of the Chinese herbal medicine QHD in the treatment of non-alcoholic fatty liver disease," Oncotarget, vol. 17, no. 8, pp. 27820-27838, 2017.

[26] C. Chang, C. Lu, C. Lin et al., "Antrodia cinnamomea reduces obesity and modulates the gut microbiota in high-fat diet-fed mice," International Journal of Obesity, vol. 6, pp. 1-45, 2017.

[27] S. Lim and D. Kim, "Bifidobacterium adolescentis IM38 ameliorates high-fat diet-induced colitis in mice by inhibiting $\mathrm{NF}-\kappa \mathrm{B}$ activation and lipopolysaccharide production by gut microbiota," Nutrition Research, vol. 41, pp. 86-96, 2017.

[28] T. Saito, H. Hayashida, and R. Furugen, "Metabolic endotoxemia initiates obesity and insulin resistance," Diabetes, vol. 56, no. 12, pp. 1761-1772, 2007.

[29] N. J. Kellow and M. T. Coughlan, "Effect of diet-derived advanced glycation end products on inflammation," Nutrition Reviews, vol. 73, no. 11, Article ID nuv030, pp. 737-759, 2015.

[30] F. Carbonero, A. C. Benefiel, A. H. Alizadeh-Ghamsari, and H. R. Gaskins, "Microbial pathways in colonic sulfur metabolism and links with health and disease," Frontiers in Physiology, vol. 3, article 448, 2012.

[31] J. Lodowska, D. Wolny, M. Jaworska-Kik, S. Kurkiewicz, Z. Dzierzewicz, and L. Wȩglarz, "The chemical composition of endotoxin isolated from intestinal strain of Desulfovibrio desulfuricans," The Scientific World Journal, vol. 2012, Article ID 647352, pp. 4-10, 2012.

[32] S. A. Bassett, W. Young, M. P. G. Barnett, A. L. Cookson, W. C. McNabb, and N. C. Roy, "Changes in composition of caecal microbiota associated with increased colon inflammation in interleukin-10 gene-deficient mice inoculated with Enterococcus species," Nutrients, vol. 7, no. 3, pp. 1798-1816, 2015.

[33] C. L. O'Brien, P. Pavli, D. M. Gordon, and G. E. Allison, "Detection of bacterial DNA in lymph nodes of Crohn's disease patients using high throughput sequencing," Gut, vol. 63, no. 10, pp. 1596-1606, 2014.

[34] W. Jiang, N. Wu, X. Wang et al., "Dysbiosis gut microbiota associated with inflammation and impaired mucosal immune function in intestine of humans with non-alcoholic fatty liver disease," Scientific Reports, vol. 5, no. 8, article 8096, 2015.

[35] D. Zhou, Q. Pan, F.-Z. Xin et al., "Sodium butyrate attenuates high-fat diet-induced steatohepatitis in mice by improving 
gut microbiota and gastrointestinal barrier," World Journal of Gastroenterology, vol. 23, no. 1, pp. 60-75, 2017.

[36] P. Louis, K. P. Scott, S. H. Duncan, and H. J. Flint, "Understanding the effects of diet on bacterial metabolism in the large intestine," Journal of Applied Microbiology, vol. 102, no. 5, pp. 1197-1208, 2007.

[37] Z. Pataky, L. Genton, L. Spahr et al., "Impact of Hypocaloric Hyperproteic Diet on Gut Microbiota in Overweight or Obese Patients with Nonalcoholic Fatty Liver Disease: A Pilot Study," Digestive Diseases and Sciences, vol. 61, no. 9, pp. 2721-2731, 2016.

[38] K. A. Kles and E. B. Chang, "Short-chain fatty acids impact on intestinal adaptation, inflammation, carcinoma, and failuree," Gastroenterology, vol. 130, supplement 1, no. 2, pp. S100-S105, 2006.

[39] W. S. Wan Saudi and M. Sjöblom, "Short-chain fatty acids augment rat duodenal mucosal barrier function," Experimental Physiology, vol. 102, no. 7, pp. 791-803, 2017.

[40] K. Kim, W. Gu, I. Lee, E. Joh, and D. Kim, "High fat dietinduced gut microbiota exacerbates inflammation and obesity in mice via the TLR4 signaling pathway," PLoS ONE, vol. 7, no. 10, Article ID e47713, 2012.

[41] P. Jegatheesan, S. Beutheu, K. Freese et al., "Preventive effects of citrulline on Western diet-induced non-alcoholic fatty liver disease in rats," British Journal of Nutrition, vol. 116, no. 2, pp. 191-203, 2016.

[42] S. Kapil, A. Duseja, B. K. Sharma et al., "Small intestinal bacterial overgrowth and toll-like receptor signaling in patients with non-alcoholic fatty liver disease," Journal of Gastroenterology and Hepatology, vol. 31, no. 1, pp. 213-221, 2016.

[43] T. Hirotani, M. Yamamoto, Y. Kumagai et al., "Regulation of lipopolysaccharide-inducible genes by MyD88 and Toll/IL-1 domain containing adaptor inducing IFN- $\beta$," Biochemical and Biophysical Research Communications, vol. 328, no. 2, pp. 383392, 2005.

[44] M. Das, D. S. Garlick, D. L. Greiner, and R. J. Davis, “The role of JNK in the development of hepatocellular carcinoma," Genes \& Development, vol. 25, no. 6, pp. 634-645, 2011.

[45] Y.-J. Zhang, Z.-L. Tian, X.-Y. Yu, X.-X. Zhao, and L. Yao, "Activation of integrin $\beta 1$-focal adhesion kinase-RasGTP pathway plays a critical role in TGF betal-induced podocyte injury," Cellular Signalling, vol. 25, no. 12, pp. 2769-2779, 2013.

[46] J.-P. Tsai, P.-C. Hsiao, S.-F. Yang et al., "Licochalcone a suppresses migration and invasion of human hepatocellular carcinoma cells through downregulation of MKK4/JNK via NF- $\kappa \mathrm{B}$ mediated urokinase plasminogen activator expression," PLoS ONE, vol. 9, no. 1, Article ID e86537, 2014.

[47] S. M. Ferolla, C. A. Couto, L. Costa-Silva et al., "Beneficial effect of synbiotic supplementation on hepatic steatosis and anthropometric parameters, but not on gut permeability in a population with nonalcoholic steatohepatitis," Nutrients, vol. 8 , no. 7 , article 397, 2016.

[48] X. D. Xu, Y. S. Sun, Q. S. Shao, J. F. Hu et al., "Effect of early enteral nutrition supplemented with glutamine on postoperative intestinalmucosal barrier function in patients with gastric carcinoma," Zhonghua Wei Chang Wai Ke Za Zhi, vol. 14, no. 6, pp. 436-439, 2011.

[49] A. B. Ribeiro, H. Giusti, A. P. Souza, C. R. Franci, and R. S. Saia, "Dexamethasone prevents lipopolysaccharide-induced epithelial barrier dysfunction in rat ileum," Shock, no. 6, pp. 116, 2017.

[50] S. Lei, T. Cheng, Y. Guo, C. Li, W. Zhang, and F. Zhi, "Somatostatin ameliorates lipopolysaccharide-induced tight junction damage via the ERK-MAPK pathway in Caco2 cells," European Journal of Cell Biology, vol. 93, no. 7, pp. 299-307, 2014.

[51] P. D. Cani, R. Bibiloni, C. Knauf et al., "Changes in gut microbiota control metabolic endotoxemia-induced inflammation in high-fat diet-induced obesity and diabetes in mice," Diabetes, vol. 57, no. 6, pp. 1470-1481, 2008.

[52] J. Du Plessis, H. Korf, J. Van Pelt et al., "Pro-inflammatory cytokines but not endotoxin-related parameters associate with disease severity in patients with NAFLD," PLOS ONE, vol. 11, no. 12, Article ID e0166048, 2016.

[53] A. Strier, D. Kravarusic, A. G. Coran et al., "The effect of elevated intra-abdominal pressure on TLR4 signaling in intestinal mucosa and on intestinal bacterial translocation in a rat," Journal of Laparoendoscopic \& Advanced Surgical Techniques, vol. 27, no. 2, pp. 211-216, 2017.

[54] A. Bein, A. Zilbershtein, M. Golosovsky, D. Davidov, and B. Schwartz, "LPS induces hyper-permeability of intestinal epithelial cells," Journal of Cellular Physiology, vol. 232, no. 2, pp. 381-390, 2017.

[55] A. Bocsik, F. R. Walter, A. Gyebrovszki et al., "Reversible opening of intercellular junctions of intestinal epithelial and brain endothelial cells with tight junction modulator peptides," Journal of Pharmaceutical Sciences, vol. 105, no. 2, pp. 754-765, 2016.

[56] C.-Z. Huang, Y.-T. Tung, S.-M. Hsia, C.-H. Wu, and G.-C. Yen, "The hepatoprotective effect of Phyllanthus emblica L. fruit on high fat diet-induced non-alcoholic fatty liver disease (NAFLD) in SD rats," Food \& Function, vol. 8, no. 2, pp. 842-850, 2017.

[57] Q. Meng, X.-P. Duan, C.-Y. Wang et al., "Alisol B 23-acetate protects against non-alcoholic steatohepatitis in mice via farnesoid X receptor activation," Acta Pharmacologica Sinica, vol. 38, no. 1, pp. 69-79, 2017.

[58] J. Wei, Y.-Z. Zhen, J. Cui et al., "Rhein lysinate decreases inflammation and adipose infiltration in $\mathrm{KK} / \mathrm{HlJ}$ diabetic mice with non-alcoholic fatty liver disease," Archives of Pharmacal Research, vol. 39, no. 7, pp. 960-969, 2016.

[59] S. Wang, X. Li, H. Guo et al., "Emodin alleviates hepatic steatosis by inhibiting sterol regulatory element binding protein 1 activity by way of the calcium/calmodulin-dependent kinase kinase-AMP-activated protein kinase-mechanistic target of rapamycin-p70 ribosomal S6 kinase signaling pathway," Hepatology Research, vol. 47, no. 7, pp. 683-701, 2016.

[60] L. Qi, Q. Fu, C. Du et al., "Amelioration of hypoxia and LPSinduced intestinal epithelial barrier dysfunction by emodin through the suppression of the NF- $\kappa \mathrm{B}$ and HIF- $1 \alpha$ signaling pathways," International Journal of Molecular Medicine, vol. 34, no. 6, pp. 1629-1639, 2014.

[61] H. Matsuda, T. Kageura, I. Toguchida, T. Murakami, A. Kishi, and M. Yoshikawa, "Effects of sesquiterpenes and triterpenes from the rhizome of Alisma orientale on nitric oxide production in lipopolysaccharide-activated macrophages: Absolute stereostructures of alismaketones-B 23-acetate and -C 23-acetate," Bioorganic \& Medicinal Chemistry Letters, vol. 9, no. 21, pp. 3081-3086, 1999.

[62] X. Tang, Z. Liao, Y. Huang, X. Lin, and L. Wu, "Atractylenolide I protects against lipopolysaccharide-induced disseminated intravascular coagulation by anti-inflammatory and anticoagulation effect," Asian Pacific Journal of Tropical Medicine, vol. 10, no. 6, pp. 582-587, 2017.

[63] M. S. Yoou, S. Y. Nam, M. H. Jin, S. Y. Lee et al., "Ameliorative effect of atractylenolide III in the mast cell proliferation induced by TSLP," Food and Chemical Toxicology, vol. 8, pp. 78-85, 2017. 


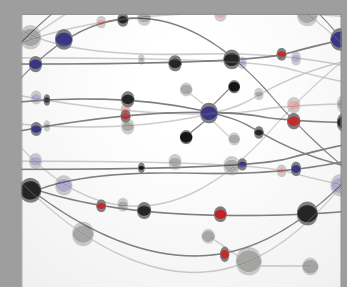

The Scientific World Journal
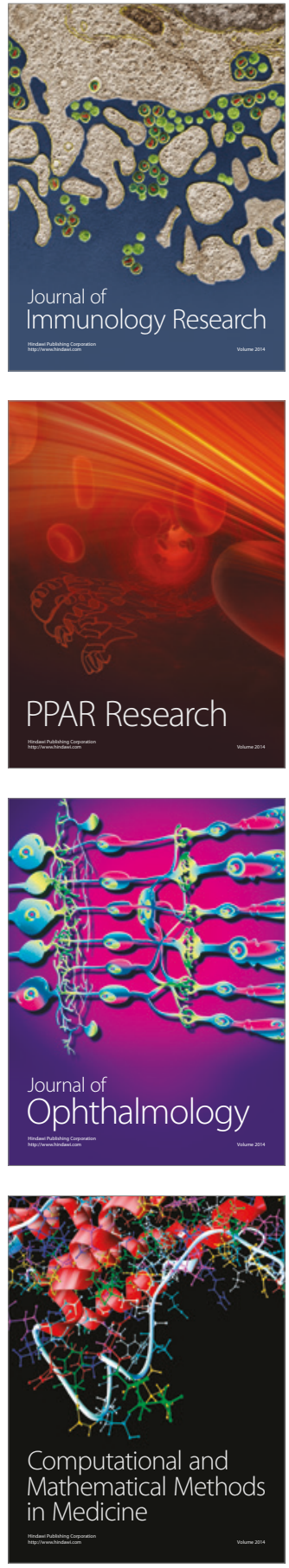

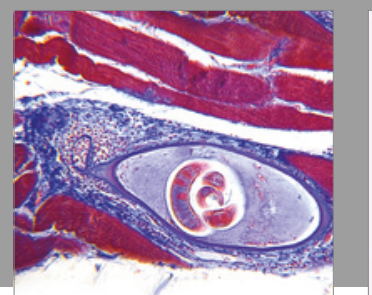

Gastroenterology Research and Practice
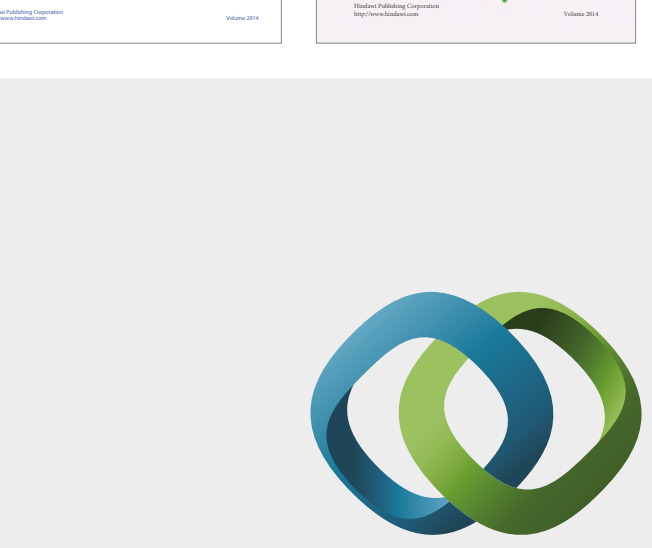

\section{Hindawi}

Submit your manuscripts at

https://www.hindawi.com
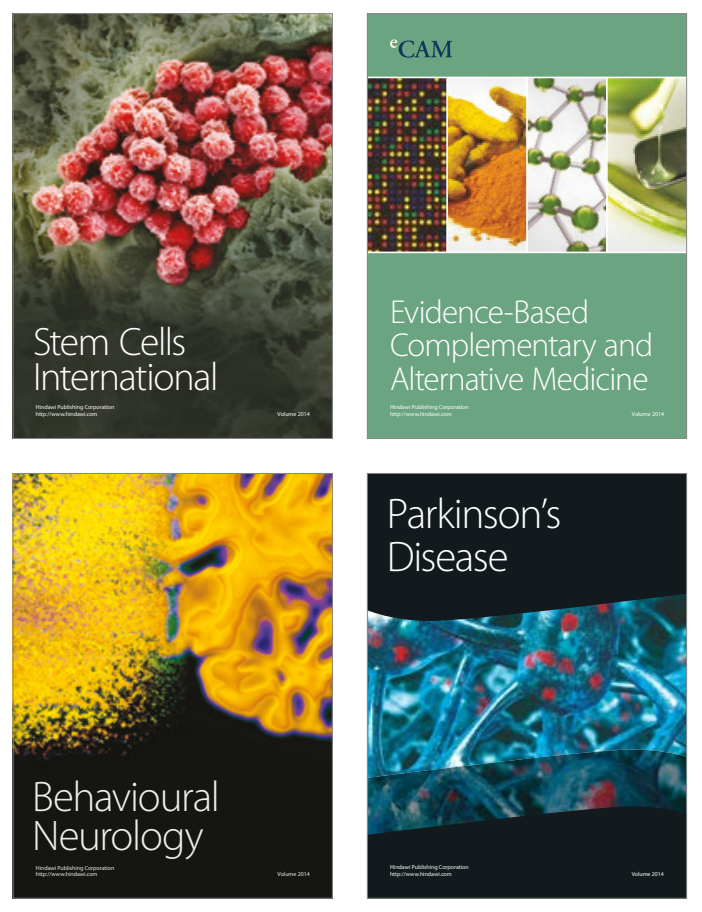
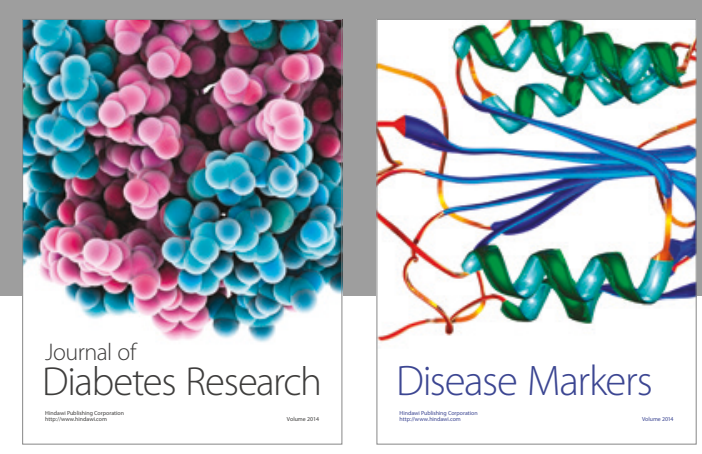

Disease Markers
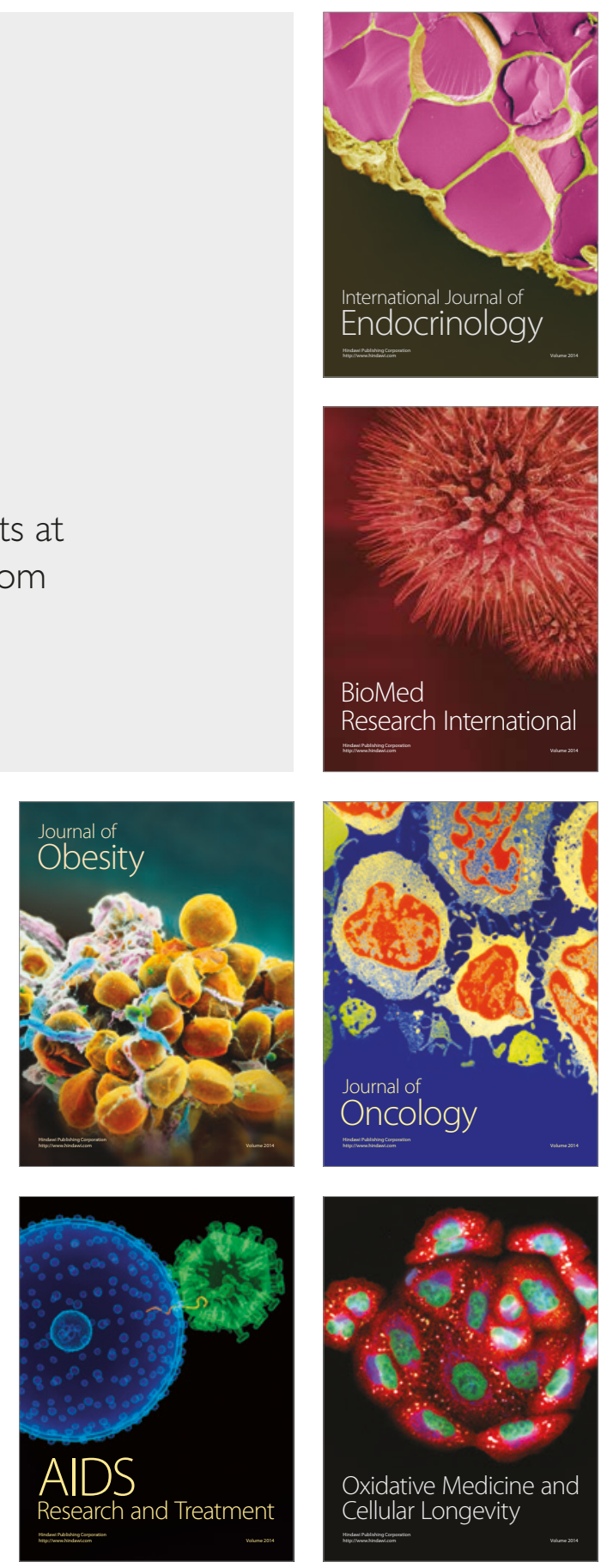